12

\title{
Аксептанс и пропускание квадрупольного фильтра масс с префильтром
}

\author{
(C) В.Б. Алмазов, ${ }^{1}$ Н.В. Коненков ${ }^{2}$ \\ ${ }^{1}$ Институт аналитического приборостроения РАН, \\ 190103 Санкт-Петербург, Россия \\ ${ }^{2}$ Рязанский государственный университет им. С.А. Есенина, \\ 390000 Рязань, Россия \\ e-mail: diamondvictor@mail.ru
}

(Поступило в Редакцию 8 ноября 2016 г. В окончательной редакции 24 марта 2017 г.)

\begin{abstract}
Предложена аналитическая модель квадрупольного фильтра масс с дополнительным радиочастотным квадруполем (префильтром) на его входе, основанная на модели переходного поля в области между торцами электродов фильтра масс и электродами префильтра. Методами динамики фазового пространства исследованы характеристики составного фильтра масс. При наличии входного префильтра вместо входной диафрагмы $y$-аксептанс увеличивается в 1.5 раза относительно $x$-аксептанса. С увеличением времени пребывания иона в краевом поле контур $y$-аксептанса имеет тенденцию к вращению на фазовой плоскости, а контур $x$-аксептанса уширяется по координате $x$. Функция пропускания с увеличением времени пребывания иона в краевом поле имеет растущий и осциллирующий характер. Результат численного эксперимента с использованием программного обеспечения SIMION 8 отличается от результата аналитического расчета на $14 \%$ для $x$-аксептанса и на $35 \%$ для $y$-аксептанса.
\end{abstract}

DOI: $10.21883 /$ JTF.2017.10.45002.2092

\section{Введение}

Квадрупольный фильтр масс (КФМ), являющийся одним из наиболее часто используемых масс-анализаторов в современной технике масс-спектрометрии, был впервые предложен Паулем [1]. Одним из факторов, ограничивающим коэффициент пропускания и разрешающую способность КФМ, является входное краевое поле $[2,3]$. Впервые для увеличения чувствительности КФМ к ионам низких энергий (к ионам больших масс) использование короткого радиочастотного (РЧ) квадруполя (или префильтра), устраняющего постоянный потенциал на входе КФМ (так называемый метод delayed DC ramp), было предложено Брубаккером [4]. Исследования $[5,6]$ подтверждают, что данный метод позволяет увеличить пропускание ионов тяжелых масс и тем самым устранить негативное влияние входного краевого поля.

Кроме того, в [7-9] приводятся доказательства того, что использование префильтра позволяет увеличить пропускание и легких ионов за счет уменьшения максимальных поперечных смещений ионов в КФМ. Это означает расширение эффективной области входной апертуры КФМ и, следовательно, пропускания КФМ в целом, за счет движения ионов внутри префильтра только в РЧ-поле.

Представляется важным знание аксептанса КФМ, модифицированного префильтром, для целей согласования анализатора и источника ионов.

Впервые расчет фазовых эллипсов КФМ и контуров пропускания при наличии входного краевого поля (без префильтра) осуществил Доусон [3]. В работе использо- валась линейная модель нарастания поля от заземленной входной диафрагмы до торцов электродов КФМ. Позже подход Доусона был развит в работе [10] на основе модели краевого поля с экспоненциально нарастающим потенциалом [11]. В [12] проводился расчет фазовых эллипсов и траекторий движения ионов для КФМ с исключенной постоянной составляющей в начальной части КФМ, но при этом также использовались линейные модели нарастания потенциала поля.

Целью настоящей работы является исследование влияния префильтра на аксептанс и пропускание КФМ и сравнение этих характеристик со случаем, когда КФМ работает без префильтра. В расчетах использовались методы динамики фазового пространства [2,12], а также предложенная ранее модель переходного поля [13].

\section{Уравнения движения ионов в КФМ с префильтром}

Структура электродов КФМ с префильтром показана на рис. 1. Префильтр представляет собой четыре коротких электрода, близко примыкающие к электродам КФМ и имеющие такую же форму профиля, как у электродов КФМ [4-9]. Мы выделяем следующие области электрического поля внутри такой электродной системы [13]: (I) входное краевое поле между заземленной входной диафрагмой и префильтром, (II) поле префильтра, достигшее стационарного значения, (III, IV) переходное поле между префильтром и КФМ, (V) поле КФМ.

В области входного краевого поля (участок I, рис. 1) происходит нарастание РЧ-составляющей поля, т. е. параметра $q_{1}$, при этом постоянная составляющая от- 


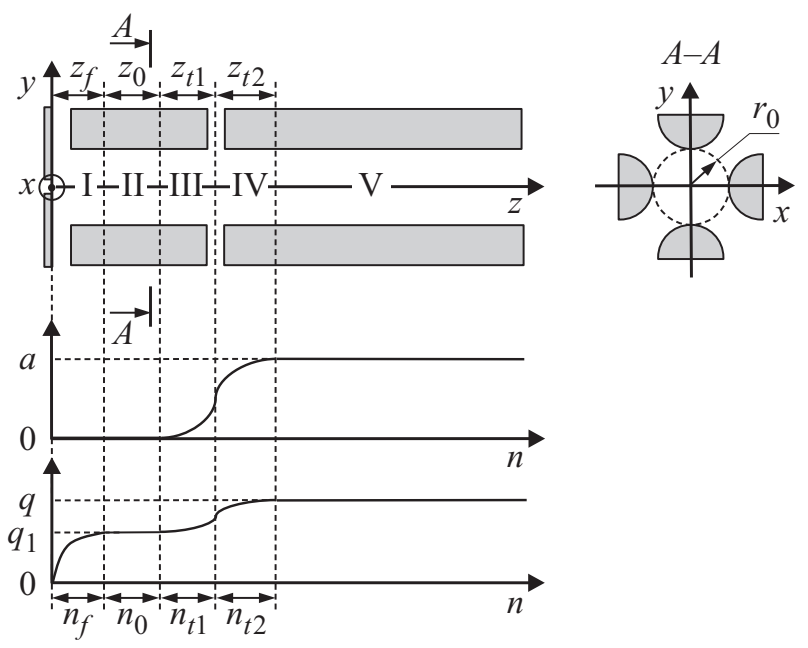

Рис. 1. Схема электродной системы КФМ с префильтром и графики изменения параметров $a, q$ : координата $z-$ расстояние, выраженное в единицах радиуса поля $r_{0}$, координата $n$ - время нахождения иона в заданной области, выраженное в количестве циклов РЧ-поля.

сутствует и параметр $a=0$. Протяженность области составляет $z_{f}$ в единицах $r_{0}$. Ион проводит в этой области $n_{f}$ циклов РЧ-поля. Используя модель краевого поля Хантера-Макинтоша [11], можно записать уравнения движения ионов в области входного краевого поля по поперечным координатам $x$ и $y$ следующим образом:

$$
\begin{aligned}
& x^{\prime \prime}-\left[2 q_{1} f(z) \cos 2\left(\xi-\xi_{0}\right)\right] x=0, \\
& y^{\prime \prime}+\left[2 q_{1} f(z) \cos 2\left(\xi-\xi_{0}\right)\right] y=0,
\end{aligned}
$$

где $z=z_{f} \xi /\left(n_{f} \pi\right)$, для $0 \leq \xi<\pi n_{f}-$ продольная координата в единицах $r_{0}, \xi=\omega t / 2-$ безразмерное время, $q_{1}=4 e V_{1} /\left(m \omega^{2} r_{0}^{2}\right)$ - параметр уравнения Матье, $e$ и $m$ - заряд и масса иона, $V_{1}$ и $\omega-$ амплитуда и круговая частота РЧ-напряжения, $r_{0}-$ радиус вписанной окружности между вершинами электродов (радиус поля [2]), $\xi_{0}$ - фаза влета иона в РЧ-поле, $f(z)=1-\exp \left(-c_{1} z-c_{2} z^{2}\right)-$ функция краевого поля Хантера-Макинтоша, описывающая нарастание потенциала вдоль оси $z, c_{1}$ и $c_{2}-$ константы, зависящие от расстояния между входной диафрагмой и торцами префильтра (рис. 1).

В области полного поля префильтра (участок II, рис. 1), протяженностью $z_{0}$, действует только РЧ-составляющая (параметр $q_{1}$ постоянен) и полностью отсутствует постоянная составляющая (параметр $a=0$ ). Уравнения движения в этой области имеют вид

$$
\begin{aligned}
& x^{\prime \prime}-\left[2 q_{1} \cos 2\left(\xi-\xi_{0}\right)\right] x=0, \\
& y^{\prime \prime}+\left[2 q_{1} \cos 2\left(\xi-\xi_{0}\right)\right] y=0
\end{aligned}
$$

на временно́м интервале $\pi n_{f} \leq \xi<\pi\left(n_{f}+n_{0}\right) ; n_{0}-$ количество периодов РЧ-поля, которое проводит ион в полном поле префильтра.
В области переходного поля между электродами префильтра и КФМ происходит постепенное нарастание постоянной составляющей поля, т.е. параметра $a$, и постепенное нарастание РЧ-составляющей, т.е. параметра $q_{1}$, до уровня $q$. Область переходного поля подразделяется на два симметричных участка (участки III, IV на рис. 1) согласно [13]. Протяженность участков в единицах радиуса поля $r_{0}$ составляет $z_{t 1}$, $z_{t 2}$ соответственно. Количества периодов РЧ-поля, в течение которого ион находится в этих участках, равны соответственно $n_{t 1}, n_{t 2}$.

Для участка III (рис. 1) уравнения движения ионов можно записать следующим образом [13]:

$x^{\prime \prime}+\left[a f_{t 1}(z)-2\left(q_{1}+\left(q-q_{1}\right) f_{t 1}(z)\right) \cos 2\left(\xi-\xi_{0}\right)\right] x=0$,

$y^{\prime \prime}-\left[a f_{t 1}(z)-2\left(q_{1}+\left(q-q_{1}\right) f_{t 1}(z)\right) \cos 2\left(\xi-\xi_{0}\right)\right] y=0$,

где $z=z_{t 1}\left[\pi\left(n_{f}+n_{0}+n_{t 1}\right)-\xi\right] /\left(n_{t 1} \pi\right)-$ продольная координата в единицах $r_{0}$ на интервале $\pi\left(n_{f}+n_{0}\right) \leq$ $\leq \xi<\pi\left(n_{f}+n_{0}+n_{t 1}\right), f_{t 1}(z)=0.5 \exp \left(-d_{1} z-d_{2} z^{2}\right)-$ функция переходного поля в конце префильтра.

Для участка IV (рис. 1) уравнения движения ионов будут иметь вид [13]

$x^{\prime \prime}+\left[a f_{t 2}(z)-2\left(q_{1}+\left(q-q_{1}\right) f_{t 2}(z)\right) \cos 2\left(\xi-\xi_{0}\right)\right] x=0$,

$y^{\prime \prime}-\left[a f_{t 2}(z)-2\left(q_{1}+\left(q-q_{1}\right) f_{t 2}(z)\right) \cos 2\left(\xi-\xi_{0}\right)\right] y=0$,

где $z=z_{t 2}\left[\xi-\pi\left(n_{f}+n_{0}+n_{t 1}\right)\right] /\left(n_{t 2} \pi\right)-$ продольная координата для $\pi\left(n_{f}+n_{0}+n_{t 1}\right) \leq \xi<\pi\left(n_{f}+n_{0}+n_{t 1}+n_{t 2}\right)$, $f_{t 2}(z)=1-0.5 \exp \left(-d_{1} z-d_{2} z^{2}\right)-$ функция переходного поля в начале КФМ.

В области действия полного поля КФМ (участок V на рис. 1) справедливо каноническое уравнение Матье $[1,2,14]$ :

$$
\begin{aligned}
& x^{\prime \prime}+\left[a-2 q \cos 2\left(\xi-\xi_{0}\right)\right] x=0, \\
& y^{\prime \prime}-\left[a-2 q \cos 2\left(\xi-\xi_{0}\right)\right] y=0
\end{aligned}
$$

для времени $\xi \geq \pi\left(n_{f}+n_{0}+n_{t 1}+n_{t 2}\right)$.

При написании уравнений движения (1)-(5) предполагается, что поля КФМ и префильтра имеют идеальное гиперболическое распределение потенциала, а скорость иона вдоль оси $z$ постоянна. Для применения уравнений (1)-(5) к реальному КФМ с префильтром необходимо учитывать, что поперечные координаты $x, y$ и скорости $x^{\prime}, y^{\prime}$ иона выражаются в безразмерных величинах, связанных с соответствующими размерными величинами следующим образом:

$$
x=X / r_{0}, y=Y / r_{0}, x^{\prime}=V_{X} /\left(\pi r_{0} f\right), y^{\prime}=V_{Y} /\left(\pi r_{0} f\right),
$$

где $X, Y$ - поперечные размерные координаты иона, $V_{X}, V_{Y}$ - поперечные размерные скорости иона, $f=$ $=\omega /(2 \pi)-$ частота РЧ-напряжения КФМ и префильтра. 


\section{Метод расчета}

Аксептанс КФМ может быть представлен контуром по заданному уровню пропускания $[10,15]$, который определяется как граница области перекрытия большого числа фазовых эллипсов, равномерно распределенных по всему диапазону начальных фаз $\xi_{0}$ на интервале $(0-\pi)$. В случае, если общее количество эллипсов равно 1000 , для уровня пропускания $100 \%$ необходимо, чтобы эта область включала в себя все 1000 эллипсов. Соответственно для определения контура по уровню пропускания 50\% область перекрытия должна включать в себя 500 эллипсов из 1000. Точность определения контура повышается с увеличением числа используемых эллипсов для начальных фаз $\xi_{0}$. Фазовый эллипс (или эллипс захвата) полного поля КФМ в одной из плоскостей $x z$ или $y z$ для одной заданной начальной фазы $\xi_{0}$ рассчитывался из уравнения [2]

$$
\Gamma u^{2}+2 A u \frac{d u}{d \xi}+B\left(\frac{d u}{d \xi}\right)^{2}=\varepsilon .
$$

Здесь $u$ - координата $x$ или $y, \varepsilon-$ площадь эллипса, деленная на $\pi$. Параметры эллипса $A, B, \Gamma$ определялись из элементов матрицы преобразования $M[2]$

$$
A=\frac{m_{11}-m_{22}}{2 \sin \pi \beta}, \quad B=\frac{m_{12}}{\sin \pi \beta}, \quad \Gamma=\frac{-m_{21}}{\sin \pi \beta},
$$

где $\beta=\arccos \left[\left(m_{11}+m_{22}\right) / 2\right] / \pi-$ параметр стабильности (характеристический показатель уравнения Матье).

Параметр $\varepsilon$ эллипса равен [2]

$$
\varepsilon=1 / B_{\max },
$$

где $B_{\max }$ - максимальное значение параметра $B\left(\xi_{0}\right)$.

Матрица преобразования $M$ рассчитывалась на интервале $\left[\pi\left(n_{f}+n_{0}+n_{t 1}+n_{t 2}\right), \pi\left(n_{f}+n_{0}+n_{t 1}+n_{t 2}+1\right)\right]$, протяженностью $\pi$, т. е. одного полного периода РЧ-поля, в области полного поля КФМ (участок V на рис. 1) как [2]:

$$
\left[\begin{array}{c}
u \\
u^{\prime}
\end{array}\right]=M\left[\begin{array}{l}
u_{0} \\
u_{0}^{\prime}
\end{array}\right]=\left[\begin{array}{ll}
m_{11} & m_{12} \\
m_{21} & m_{22}
\end{array}\right] \cdot\left[\begin{array}{l}
u_{0} \\
u_{0}^{\prime}
\end{array}\right] .
$$

Здесь $u_{0}, u_{0}^{\prime}$ - поперечные координата и скорость в начале полного поля КФМ, $u, u^{\prime}$ - поперечные координата и скорость после одного периода полного поля КФМ. Для определения элементов матрицы преобразования $M$ необходимо задаться двумя парами начальных условий: $u_{0}=0, u_{0}^{\prime}=1$ и $u_{0}=1, u_{0}^{\prime}=0$. Для расчета матрицы преобразования $M$ интегрировались численно уравнения движения ионов (5).

Фазовый эллипс полного поля КФМ модифицируется краевым, полным и переходным полями префильтра. Уравнение модифицированного эллипса имеет вид [2]

$$
\Gamma_{a} u^{2}+2 A_{a} u \frac{d u}{d \xi}+B_{a}\left(\frac{d u}{d \xi}\right)^{2}=\varepsilon .
$$

Параметры модифицированного эллипса $A_{a}, B_{a}, \Gamma_{a}$ определялись следующим образом $[2,10]$ :

$$
\left[\begin{array}{c}
B_{a} \\
A_{a} \\
\Gamma_{a}
\end{array}\right]=\left[\begin{array}{ccc}
S^{\prime 2} & 2 S S^{\prime} & S^{2} \\
C^{\prime} S^{\prime} & C^{\prime} S+S^{\prime} C & C S \\
C^{\prime 2} & 2 C C^{\prime} & C^{2}
\end{array}\right] \cdot\left[\begin{array}{c}
B \\
A \\
\Gamma
\end{array}\right] .
$$

Элементы $S, S^{\prime}, C, C^{\prime}$ матрицы преобразования $M_{f}$ рассчитывались на интервале $\left[0, \pi\left(n_{f}+n_{0}+n_{t 1}+n_{t 2}\right)\right]$ путем численного интегрирования уравнений движения ионов (1)-(4) [10]:

$$
\left[\begin{array}{l}
u_{e} \\
u_{e}^{\prime}
\end{array}\right]=M_{f}\left[\begin{array}{l}
u_{a} \\
u_{a}^{\prime}
\end{array}\right]=\left[\begin{array}{ll}
C & S \\
C^{\prime} & S^{\prime}
\end{array}\right] \cdot\left[\begin{array}{l}
u_{a} \\
u_{a}^{\prime}
\end{array}\right] .
$$

Здесь $u_{e}, u_{e}^{\prime}$ - поперечные координата и скорость в начале полного поля КФМ, $u_{a}, u_{a}^{\prime}$ - поперечные координата и скорость в начале префильтра. Способ расчета матрицы преобразования $M_{f}$ аналогичен способу расчета матрицы $M$ : используются те же начальные условия $u_{a}=0, u_{a}^{\prime}=1$ и $u_{a}=1, u_{a}^{\prime}=0$.

Коэффициент пропускания $T$ КФМ определялся путем расчета траекторий движения ионов в КФМ при различных начальных условиях (начальных координатах $x_{0}, y_{0}$, скоростях $x_{0}^{\prime}, y_{0}^{\prime}$ и фаз $\left.\xi_{0}\right)$ как

$$
T=N_{\mathrm{tr}} / N,
$$

где $N$ - общее число рассчитываемых траекторий, $N_{\mathrm{tr}}$ - число траекторий, которые имеют амплитуду колебаний менее радиуса поля $r_{0}$. Траектории движения ионов рассчитывались путем интегрирования уравнений движения ионов (1)-(5) на интервале времени от 0 до $n_{\text {all }} \mathcal{T}$, где $n_{\text {all }}$ - общее число периодов РЧ-поля, в течение которых ион находился в КФМ. Начальные координаты $x_{0}, y_{0}$ и скорости $x_{0}^{\prime}, y_{0}^{\prime}$ назначались случайным образом с нормальным законом распределения на интервале поперечных координат от -0.4 до 0.4 и интервале поперечных скоростей от -0.05 до 0.05. Для каждой выбранной таким образом точки $\left(x_{0}, y_{0}, x_{0}^{\prime}, y_{0}^{\prime}\right)$ фазового пространства выполнялся перебор начальных фаз $\xi_{0}$ в диапазоне $(0-\pi)$.

Для решения уравнений движения ионов (1)-(5) применялся численный метод Рунге-Кутта-Нюстрема-Дорманда-Принса шестого порядка [16].

В расчетах использовались следующие значения параметров краевого и переходного полей: $z_{f}=z_{t 1}=z_{t 2}=1.5$, $c_{1}=2.15, c_{2}=1.77, d_{1}=2.56, d_{2}=1.70$ [13]. Рабочая точка на диаграмме стабильности имела координаты: $a=0.23343, q=0.706$, что соответствует разрешающей способности $R=50$ [2]. Параметр $q_{1}$ был выбран равным $0.5 q$, т.е. амплитуда РЧ-напряжения $V_{1}$ на электродах префильтра в 2 раза меньше, чем на электродах КФМ.

Для определения времен $n_{f}, n_{0}, n_{t 1}, n_{t 2}$ нахождения иона на участках I-V (рис. 1) и общего времени пребывания иона в электродной системе $n_{\text {all }}$ предполагалось, 

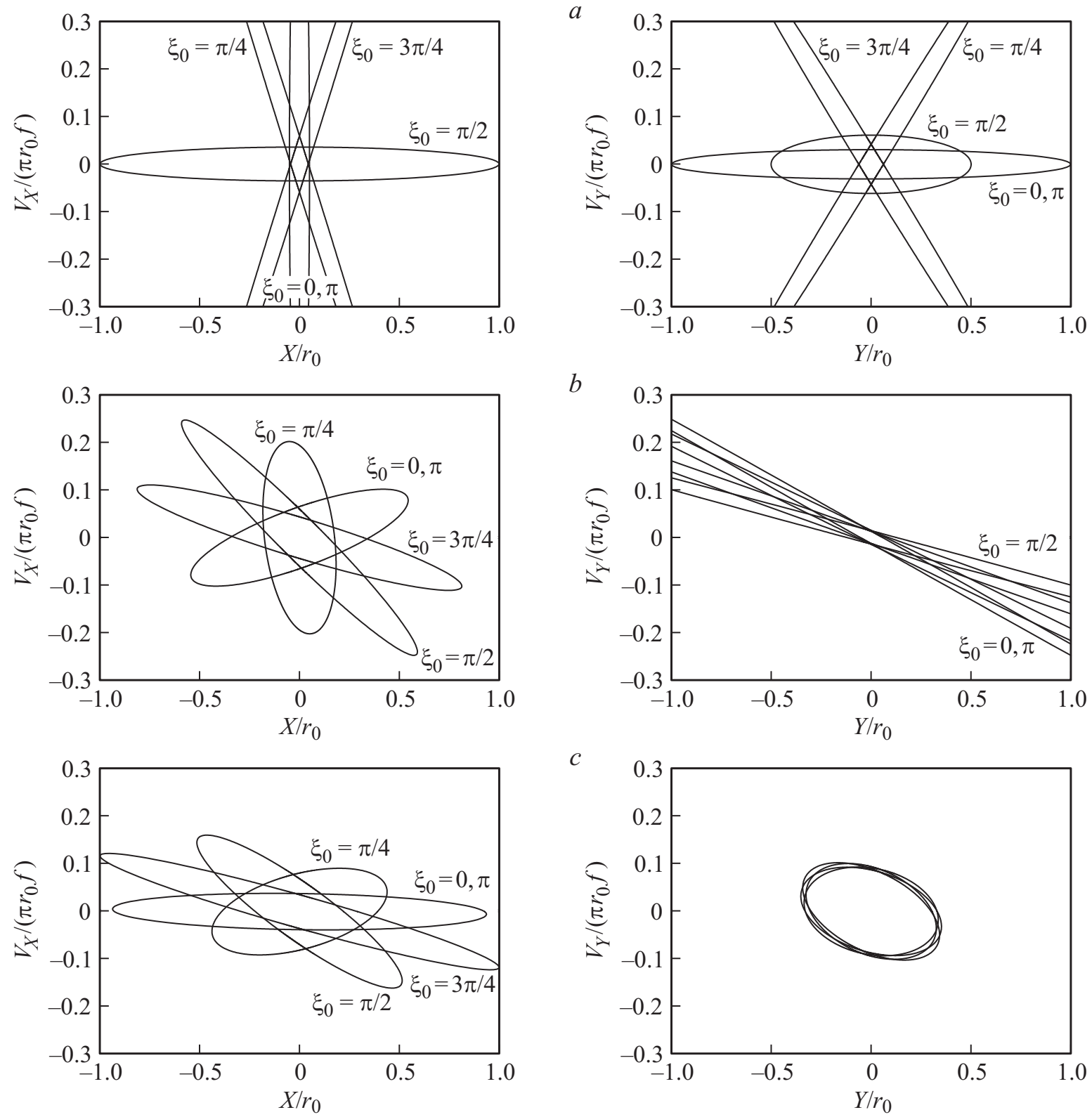

Рис. 2. Эллипсы захвата для $x$ и $y$-координат. $R=50, M=500, E=7 \mathrm{eV}\left(n_{f}=5.347\right)$; $a$ - краевое поле КФМ отсутствует, $b-$ КФМ с входной диафрагмой, $c-$ КФМ с префильтром.

что КФМ с префильтром имеет следующие параметры: частота РЧ-напряжения $f=1.1 \mathrm{MHz}$, радиус поля $r_{0}=5.325 \mathrm{~mm}$, длина префильтра $L_{p}=3.76 r_{0}$, длина КФМ $L_{q}=25 r_{0}$, зазор между входной диафрагмой и префильтром $d=0.25 r_{0}$, зазор между префильтром и КФМ $h=0.25 r_{0}$.

В случае расчета характеристик КФМ с входной диафрагмой параметры КФМ оставались теми же, и использовалась методика расчета, описанная в [10]. Протяженность области краевого поля в таком случае составляет $z_{f}=1.5$ в единицах $r_{0}$, ион проводит в этой области $n_{f}$ периодов РЧ-поля. В расчетах характеристик КФМ с входной диафрагмой применялась экспоненциальная модель краевого поля Хантера-Макинтоша [11].

\section{Результаты расчета}

На рис. 2 для сравнения приведены фазовые эллипсы КФМ без учета краевого поля [2,14], КФМ с входной диафрагмой [10] и КФМ с префильтром в $x$-, $y$-направлении для четырех начальных фаз при одних и тех же условиях. Краевое поле КФМ приводит к совмещению фазовых эллипсов как в $x$ - так и в $y$-направлении, т.е. условия ввода ионов становятся близкими для разных фаз. Эллипсы наклоняются влево, т. е. сходящийся входной пучок ионов становится наиболее предпочтительным для всех начальных фаз. Подобные особенности фазовых эллипсов КФМ с входной диафрагмой отмечались также в $[9,12]$. 

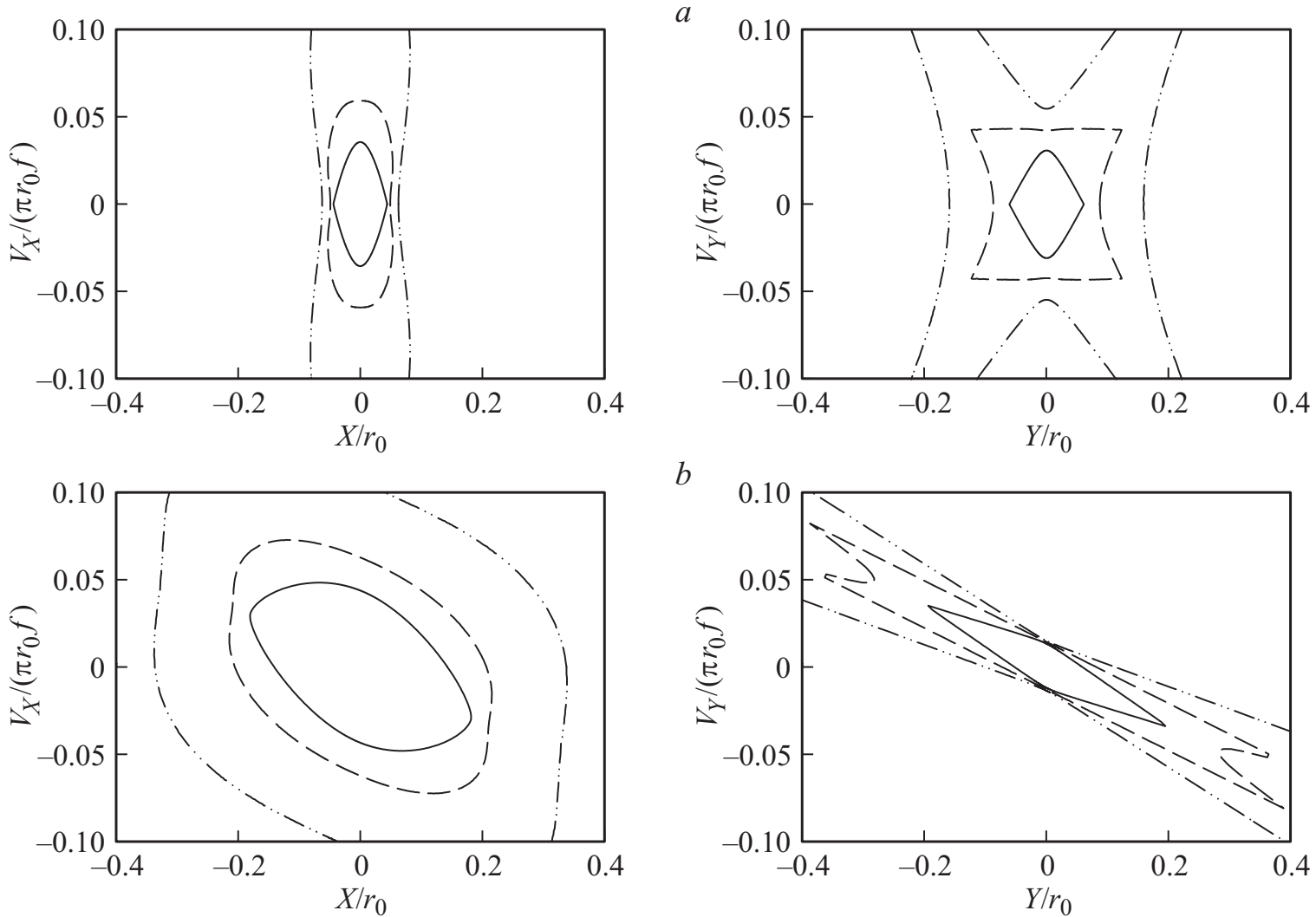

$b$
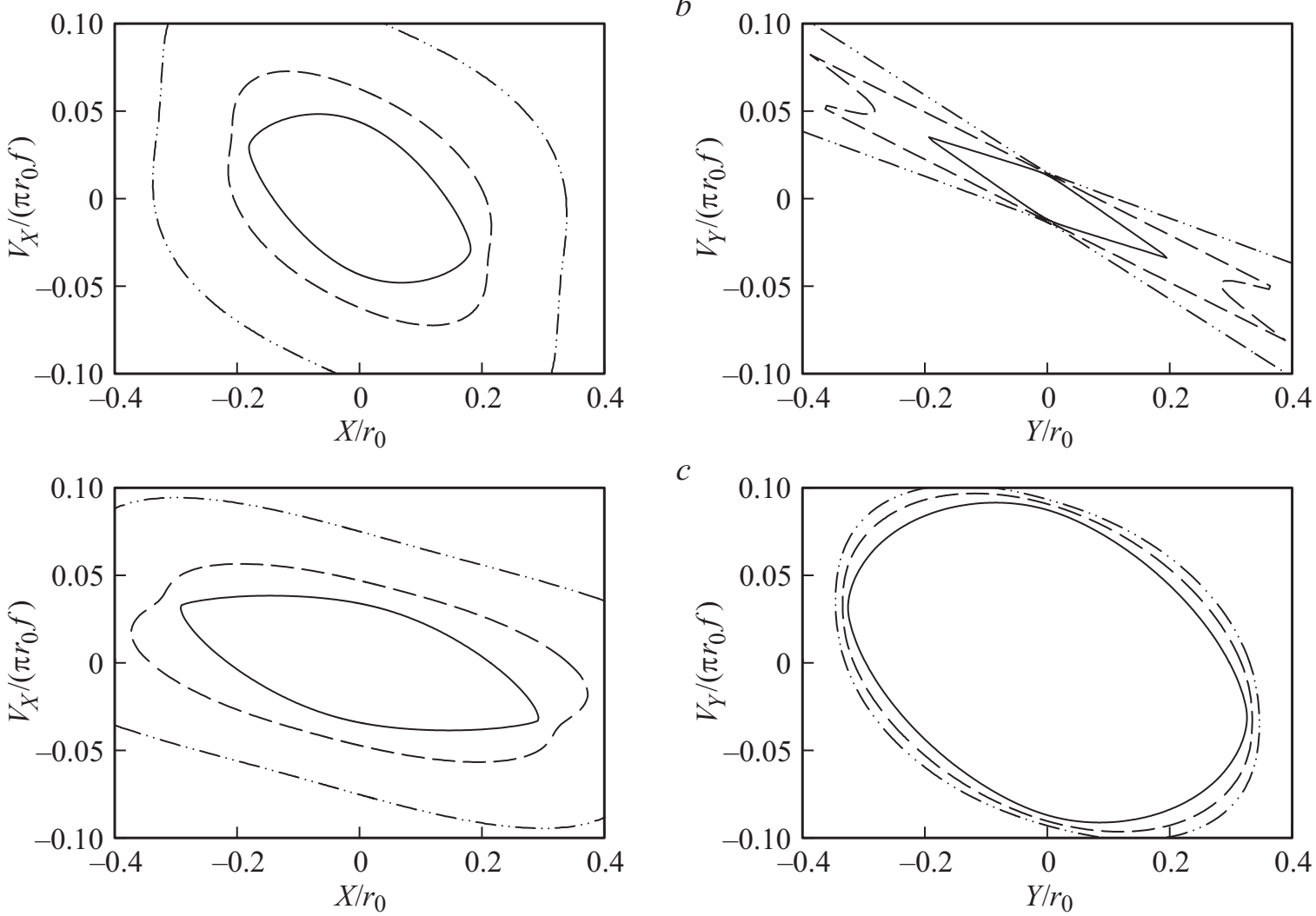

Рис. 3. Контуры пропускания на фазовых плоскостях начальных поперечных координат и скоростей: —— уровень пропускания $100 \%,---$ уровень пропускания $50 \%,-\cdots-$ уровень пропускания $25 \% . R=50, M=500, E=7 \mathrm{eV}\left(n_{f}=5.347\right) ; a-$ контуры пропускания КФМ без учета краевого поля, $b$ - контуры пропускания КФМ с входной диафрагмой, $c-$ контуры пропускания КФМ с префильтром.

Совмещение фазовых эллипсов также имеет место в случае КФМ с префильтром и особенно сильно проявляется в $y$-направлении, где эллипсы совпадают почти полностью. Такую особенность $y$-направления КФМ с префильтром можно объяснить влиянием протяженного РЧ-поля префильтра, переходного поля между префильтром и КФМ, а также действием отрицательного постоянного потенциала КФМ на положительный ион в $y$-направлении.

На рис. 3 показаны аксептансы в виде контуров пропускания по уровням 25, 50, 100\% для КФМ без учета краевого поля $(a)$, КФМ с входной диафраг- мой $(b)$ и КФМ с префильтром $(c)$. Каждый контур определялся с использованием 1000 эллипсов захвата (рис. 2). Контуры на фазовой плоскости начальных координат и скоростей представляют собой диаграммы распределения вероятностей пролета ионов через КФМ. Краевое поле КФМ увеличивает $x$-аксептанс, но уменьшает $y$-аксептанс. Увеличение $x$-аксептанса обусловлено совмещением фазовых эллипсов $x$-направления в КФМ с входной диафрагмой (рис. 2,b). Уменьшение $y$-аксептанса объясняется тем, что при прохождении ионом краевого поля параметры стабильности $a, q$ уравнения Матье на некоторое время оказы- 
ваются вне области стабильных траекторий в $y z$-плоскости КФМ [4].

Наличие префильтра незначительно изменяет $x$-аксептанс, но существенно увеличивает $y$-аксептанс. Контуры по уровням пропускания 25, 50, и 100\% y-аксептанса почти совпадают (рис. 3,c). Таким образом, аксептанс КФМ с префильтром в $y$-направлении становится более сосредоточенным, чем в $x$-направлении, и его величина по уровням пропускания 50, 100\% превышает величину аксептанса в $x$-направлении по тем же уровням пропускания. Это вызвано тем, что в $y$-направлении фазовые эллипсы КФМ с префильтром близки к совпадению (рис. 2,c). В результате следует ожидать увеличения коэффициента пропускания $T$.

Вышеприведенные результаты расчетов фазовых эллипсов и контуров пропускания были получены при следующих длительностях нахождения иона в различных областях КФМ с префильтром (рис. 1): $n_{f}=5.347$, $n_{0}=4.031, n_{t 1}=5.347, n_{t 2}=5.347$, что соответствует движению иона массой $M=500$ при транспортной энергии $E=7 \mathrm{eV}$ с продольной скоростью $0.28053 r_{0} /$ период.

Для оценки пропускания КФМ в зависимости от продольной скорости движения иона (или транспортной энергии иона) рассчитана зависимость произведения площадей фигур, ограниченных контурами на фазовых плоскостях от времени $n_{f}$ пребывания иона в краевой области, выраженного в числе периодов РЧ-поля. На рис. 4, 5 представлены зависимости безразмерных площадей областей $S_{x}, S_{y}$, ограниченных контурами пропускания по уровню $100 \%$, и их произведений $S_{x} S_{y}$ от количества периодов РЧ-поля $n_{f}$ для случаев КФМ с входной диафрагмой и КФМ с префильтром. Величины размерных площадей $S_{X}, S_{Y}$ и их произведение $S_{X} S_{Y}$ связаны с безразмерными величинами $S_{x}, S_{y}, S_{x} S_{y}$ следующими соотношениями:

$$
S_{X}=S_{x} \pi r_{0}^{2} f, \quad S_{Y}=S_{y} \pi r_{0}^{2} f, \quad S_{X} S_{Y}=S_{x} S_{y} \pi^{2} r_{0}^{4} f^{2}
$$

В КФМ с входной диафрагмой (рис. 4) с увеличением времени $n_{f} y$-аксептанс уменьшается, в то время как $x$-аксептанс, осциллируя с периодом 2.5 периода поля, возрастает. При $n_{f}=2 y$-аксептанс достигает максимума и далее монотонно убывает. Произведение площадей $S_{x} S_{y}$ (комбинированный аксептанс) достигает максимума при $n_{f} \approx 3.8$. Уменьшение $y$-аксептанса с увеличением $n_{f}$ выше этого значения приводит к снижению комбинированного аксептанса $S_{x} S_{y}$ а значит к дискриминации тяжелых (или медленных) ионов в КФМ.

При наличии префильтра (рис. 5) зависимости $S_{x}\left(n_{f}\right)$ и $S_{y}\left(n_{f}\right)$ имеют ярко выраженный осциллирующий растущий характер. Периодичность осцилляций для $y$-аксептанса составляет около 2 периодов РЧ-поля, а для $x$-аксептанса около $1 / 3$ периода РЧ-поля. При $n_{f}=2$ $y$-аксептанс резко возрастает до максимального уровня $S_{y}=0.086$. В результате комбинированный аксептанс $S_{x} S_{y}$ также периодичен и имеет тенденцию к возрастанию с ростом $n_{f}$. При любом значении $n_{f}$ в диапазоне

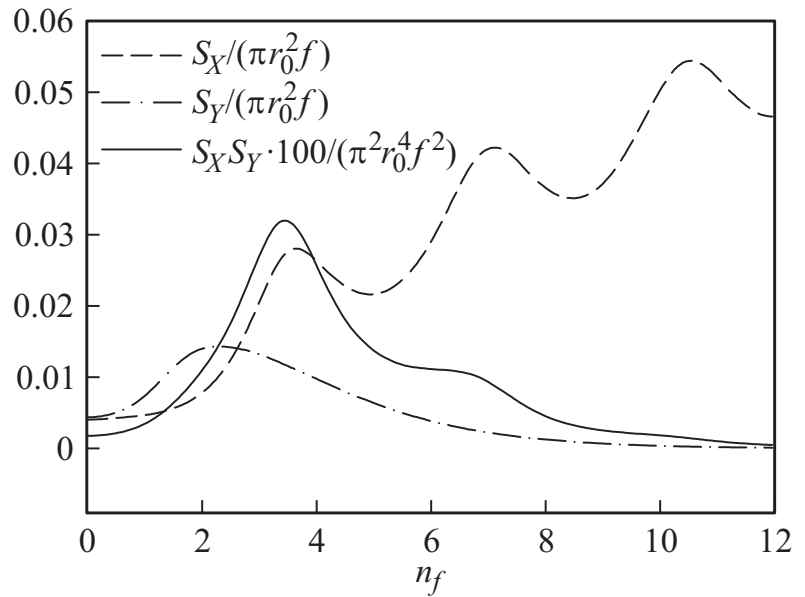

Рис. 4. Зависимости площадей $S_{x}$ и $S_{y}$, ограниченных контурами пропускания по уровню $100 \%$, и их произведения $S_{x} S_{y}$ от времени $n_{f}$ пребывания иона в краевой области для КФМ с входной диафрагмой; $R=50$.

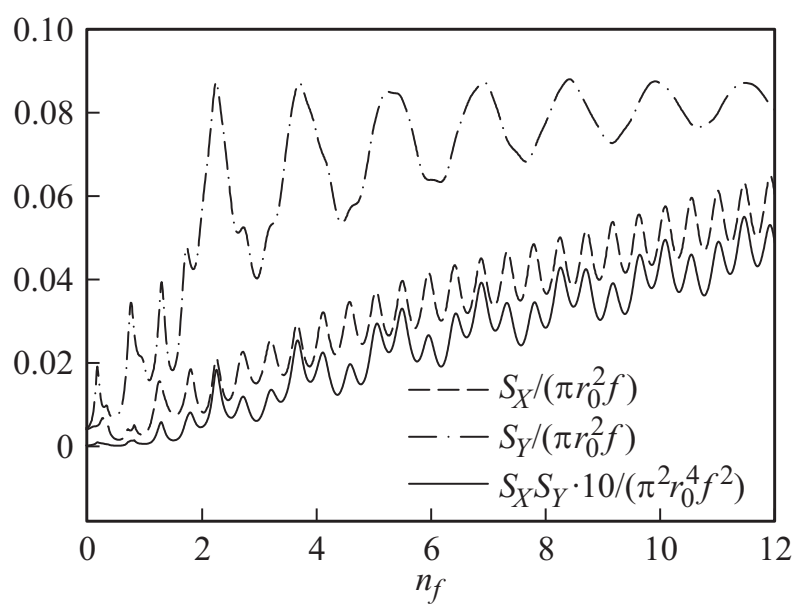

Рис. 5. Зависимости площадей $S_{x}$ и $S_{y}$, ограниченных контурами пропускания по уровню $100 \%$, и их произведения $S_{x} S_{y}$ от времени $n_{f}$ пребывания иона во входной краевой области для КФМ с префильтром; $R=50$.

от 0 до $12 y$-аксептанс в 1.5 и более раз больше $x$-аксептанса. Эту особенность поведения $S_{y}\left(n_{f}\right)$ следует учитывать при конструировании источника ионов, когда необходимо, чтобы эмиттанс источника ионов в наибольшей степени соответствовал аксептансу КФМ для обеспечения максимального коэффициента пропускания анализатора.

Комбинированный аксептанс $S_{x} S_{y}$ для КФМ с входной диафрагмой (рис. 4) имеет максимум около 0.0003 при $n_{f} \approx 3.5$. Произведение $S_{x} S_{y}$ для КФМ с префильтром для того же значения $n_{f}$ равно примерно 0.002 , что почти на порядок больше.

На рис. 6 приведены контуры пропускания по уровню $100 \%$ КФМ с префильтром для различных значений времени $n_{f}$. Контуры для $y$-направления имеют тенденцию к вращению и изменению площади ограниченной ими 
области, что необходимо учитывать при согласовании источника ионов и КФМ с префильтром. Контуры для $x$-направления с увеличением $n_{f}$ расширяются по координате, увеличивая ограниченную ими область.
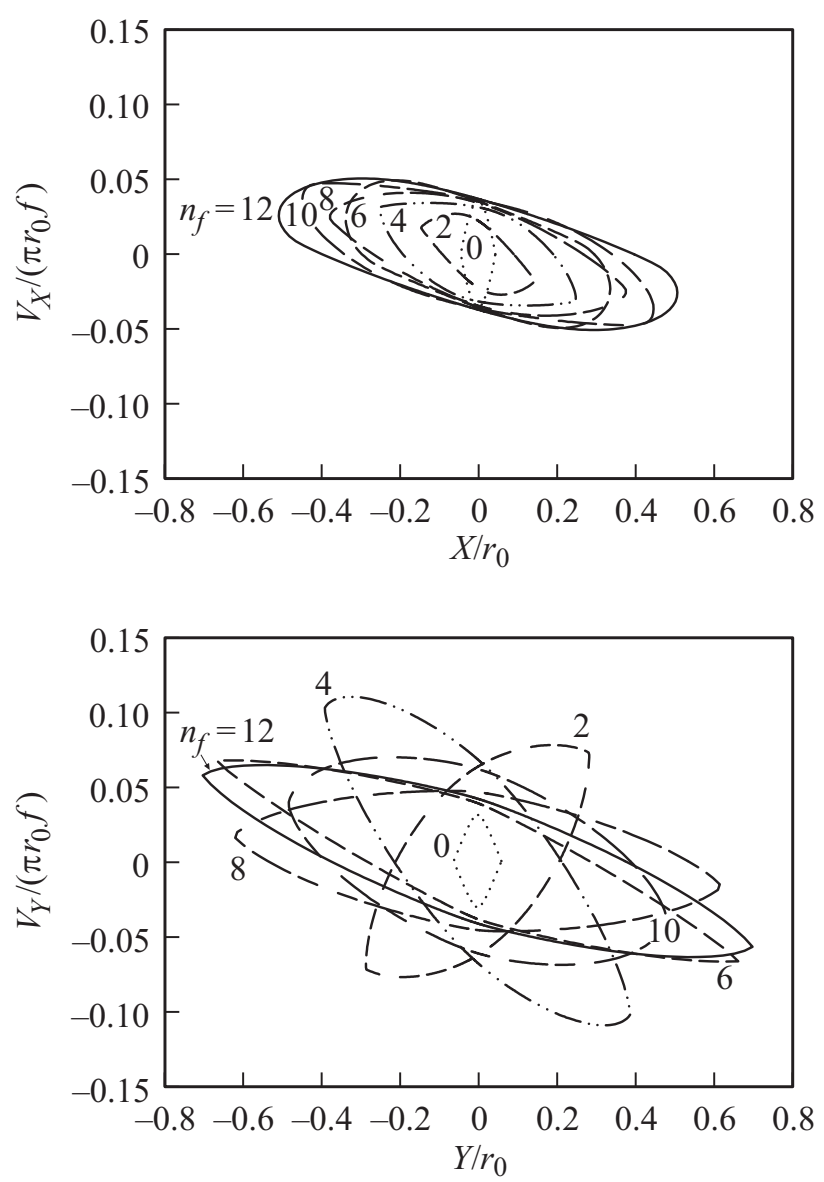

Рис. 6. Влияние времени $n_{f}$ пребывания иона во входной краевой области на контуры пропускания по уровню $100 \%$ для КФМ с префильтром; $R=50$.

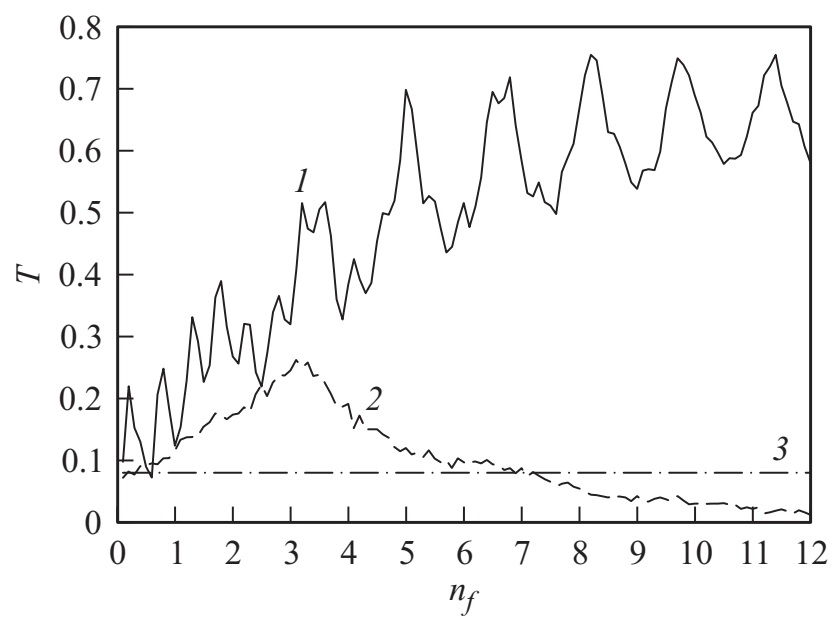

Рис. 7. Зависимости коэффициента пропускания $T$ от времени $n_{f}$ пребывания ионов во входной краевой области: $1-$ КФМ с префильтром, $2-$ КФМ с входной диафрагмой, 3 - уровень $T$ для КФМ без учета краевого поля; $R=50$.
В реальном устройстве коэффициент пропускания КФМ будет зависеть не только от изменения площадей, ограниченных контурами пропускания, но и от конфигурации эмиттанса источника ионов. На рис. 7 приведены зависимости коэффициента пропускания $T$ от времени $n_{f}$ пребывания ионов во входном краевом поле (для случаев КФМ с входной диафрагмой и КФМ с префильтром). Характер изменения коэффициента пропускания $T$ (рис. 7) хорошо согласуется с характером изменения произведения площадей фигур, ограниченных контурами пропускания (рис. 4, 5).

При $n_{f}<7$, КФМ с входной диафрагмой дает повышение коэффициента пропускания $T$ в сравнении со случаем, когда не учитывается краевое поле. Входное краевое поле можно не принимать во внимание при больших продольных скоростях ионов или при малом времени пребывания ионов в переходном и краевом полях $\left(n_{f} \rightarrow 0\right)$. Коэффициент пропускания $T$ КФМ с префильтром значительно превышает $T$ КФМ с входной диафрагмой при любом $n_{f}$.

Для данного модельного источника ионов общий вид и периодичность коэффициента пропускания $T$ КФМ с префильтром близки к виду и периодичности зависимости $y$-аксептанса от $n_{f}$, а не произведения $x$ - и $y$-аксептансов, что можно объяснить большим влиянием $y$-аксептанса на трансмиссию, из-за его значительно большего значения по сравнению с $x$-аксептансом.

Как видно из рис. 7, пропускание КФМ с префильтром ионов низких энергий возрастает, однако на практике с уменьшением транспортной энергии интенсивность массовых пиков падает. Это объясняется тем, что эффективность работы источника ионов при энергиях, меньших порядка $2 \mathrm{eV}$, резко падает из-за проявления пространственного заряда, наличия диэлектрических пленок на электродах и дефектов сборки электродов. Таким образом, для повышения чувствительности КФМ с префильтром основной задачей является создание эффективного источника ионов низких энергий. Кроме того, если рис. 7 рассматривать как график трансмиссии для ионов различных масс (который был построен при постоянной разрешающей способности $R=50$ на всем диапазоне изменения $n_{f}$ ), то можно также видеть, что префильтр позволит до некоторой степени компенсировать падение трансмиссии в случае увеличения разрешающей способности в области высоких масс.

\section{Численный эксперимент}

Для проверки предложенной аналитической модели КФМ с префильтром, с помощью программного обеспечения SIMION 8 [17] была создана численная модель, состоящая из электродов КФМ и префильтра гиперболического профиля. Параметры модели были следующими: массовое число $M=500$, разрешение $R=50$, количество начальных фаз РЧ-напряжения 30 , энергия ионов $7 \mathrm{eV}$, частота РЧ-напряжения $f=1.1 \mathrm{MHz}$, длина КФМ 

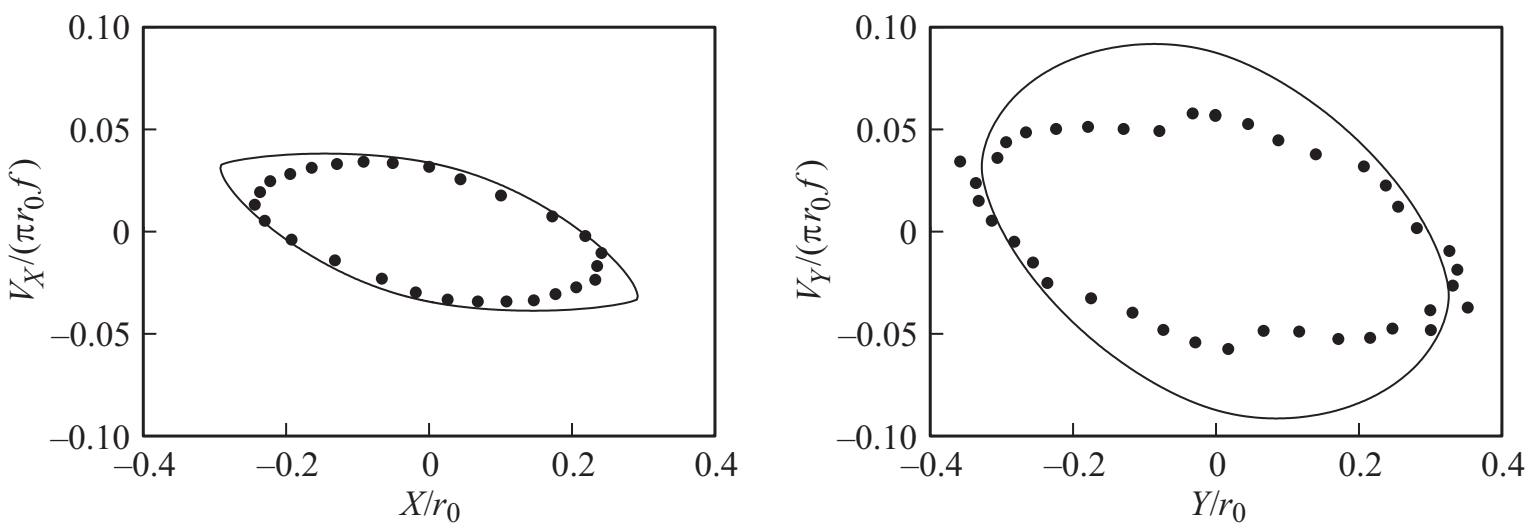

Рис. 8. Контуры пропускания по уровню $100 \%$ КФМ с префильтром: …… контуры, полученные в результате численного моделирования, — _онтуры, полученные путем аналитического расчета.

$L_{q}=131 \mathrm{~mm}$, длина префильтра $L_{p}=20 \mathrm{~mm}$, зазор между КФМ и префильтром $h=1 \mathrm{~mm}$, радиус вписанной окружности $r_{0}=5.3125 \mathrm{~mm}$. Точность представления КФМ $-0.00625 \mathrm{~mm}$ на точку, префильтра $-0.0625 \mathrm{~mm}$ на точку.

На рис. 8 показаны контуры пропускания по уровню $100 \%$, определенные с помощью численной SIMIONмодели, и контуры пропускания, рассчитанные с использованием предложенной аналитической модели. Площади областей, ограниченных полученными контурами, следующие: для аналитической модели $S_{X}=0.028 \pi r_{0}^{2} f, S_{Y}=0.085 \pi r_{0}^{2} f$, для численной модели $-S_{X}=0.024 \pi r_{0}^{2} f, S_{Y}=0.055 \pi r_{0}^{2} f$.

По результатам численного моделирования площадь, ограниченная контуром $y$-аксептанса, больше площади, ограниченной контуром $x$-аксептанса, так же как и по результатам аналитического расчета, что говорит о соблюдении в SIMION-модели ранее выявленных закономерностей аналитической модели. Контуры, рассчитанные аналитически и полученные в результате моделирования, имеют схожие формы и близкие размеры.

Отличия между аналитически рассчитанными и полученными в результате численного моделирования контурами наблюдаются в области максимальных координат $x$-направления и в области максимальных поперечных скоростей $y$-направления, что вызвано факторами, обусловленными идеализациями при выполнении аналитического расчета и неточностями численной SIMION-модели. Таковыми факторами являются следующие. В аналитическом расчете: (i) использование упрощенных одномерных моделей краевого и переходного полей, не учитывающих характер поля в приэлектродных областях; (ii) независимость расчетных траекторий движения ионов в $x$ - и $y$-направлениях, что также является следствием использования упрощенных одномерных моделей краевого и переходного полей; (iii) отсутствие $z$-составляющей поля в используемых упрощенных одномерных моделях краевого и переходного полей, что означает постоянство скорости движения иона вдоль оси $z$. В SIMION-модели: (i) конечность гиперболических электродов в поперечных направлениях; (ii) представление электродов в программе SIMION в виде конечного набора дискретных элементов. Факторы идеализации аналитического расчета являются, очевидно, наиболее значимыми.

\section{Заключение}

В настоящей работе предложен способ расчета фазовых эллипсов и аксептанса КФМ с префильтром методами динамики фазового пространства с использованием аналитической модели переходного поля в области между префильтром и КФМ.

Расчет показывает, что наличие префильтра приводит к почти полному совпадению фазовых эллипсов в $y$-направлении, в результате $y$-аксептанс по уровню пропускания $100 \%$ в рассмотренном случае становится не менее чем в 1.5 раза больше $x$-аксептанса по тому же уровню пропускания. Контуры аксептанса в $y$-направлении имеют тенденцию к вращению при изменении времени $n_{f}$ пребывания иона во входном краевом поле, что означает изменение требований к характеру входного пучка ионов. Так, при $n_{f}=2$ предпочтителен расходящийся входной пучок ионов, а при $n_{f}=4-$ сходящийся. Контуры аксептанса в $x$-направлении с возрастанием времени $n_{f}$ увеличиваются по координате до $0.5 r_{0}$ при $n_{f}=12$, при этом предпочтительный входной пучок ионов остается сходящимся.

Функция коэффициента пропускания $T$ КФМ с префильтром при постоянной разрешающей способности $R=50$ с увеличением времени $n_{f}$ пребывания иона во входном краевом поле от 0 до 12 периодов имеет возрастающий и периодический характер, с периодичностью около двух периодов РЧ-поля. Аксептанс по уровню пропускания $100 \%$ в $x$-направлении в этом диапазоне времени $n_{f}$ равномерно возрастает, а аксептанс по уровню пропускания $100 \%$ в $y$-направлении при $n_{f}=2$ резко возрастает до предельного значения $S_{Y}=0.086 \pi r_{0}^{2} f$. 
Численный эксперимент с использованием программного обеспечения SIMION 8 свидетельствует об адекватности предложенной модели переходного поля и метода расчета аксептанса КФМ с префильтром, несмотря на наличие идеализаций в модели и в методе расчета.

\section{Список литературы}

[1] Paul W., Steinwedel $H$. // Zeitschrift für Naturforschung. 1953. 8A. P. 448-450.

[2] Dawson P.H. Quadrupole Mass Spectrometry and its Applications. N.Y: American Institute of Physics, 1995. $377 \mathrm{p}$.

[3] Dawson P.H. // Int. J. Mass Spectrom. 1975. Vol. 17. N 4. P. 423-445.

[4] Brubaker W.M. // Adv. Mass Spectrom. 1968. Vol. 4. P. 293-299.

[5] Wright S., O'Prey S., Syms R., Hong G., Holmes A. // J. Microelectromech. Syst. 2010. Vol. 19. N 2. P. 325-337.

[6] Arnold W. // J. Vac. Sci. Technol. 1970. Vol. 7. N 1. P. 191-194.

[7] Trajber C., Simon M., Csatlos M. // Meas. Sci. Technol. 1991. Vol. 2. N 8. P. $785-787$.

[8] Trajber C., Simon M., Bohatka S. // Rapid Commun. Mass Spectrom. 1992. Vol. 6. N 7. P. 459-462.

[9] Dawson P.H. // Int. J. Mass Spectrom. 1971. Vol. 6. P. 33-44.

[10] Mc Intosh B.J., Hunter K.L. // Int. J. Mass Spectrom. 1989. Vol. 87. P. $165-179$.

[11] Hunter K.L., Mc Intosh B.J. // Int. J. Mass Spectrom. 1989. Vol. 87. P. $157-164$.

[12] Dawson P.H. // Int. J. Mass Spectrom. 1977. Vol. 25. P. $375-392$.

[13] Алмазов В.Б. // Научное приборостроение. 2016. Т. 26. № 3. C. $75-82$.

[14] Слободенюк Г.И. Квадрупольные масс-спектрометры. М.: Атомиздат, 1974. $272 \mathrm{c}$.

[15] Коненков Н.В., Махмудов М.Н., Страшнов Ю.В. // ЖТФ. 2009. Т. 79. Вып. 5. С. 95-100.

[16] Хайрер Э., Нерсет С., Ваннер Г. Решение обыкновенных дифференциальных уравнений. Нежесткие задачи. М.: Мир, 1990. 512 c.

[17] Manura D.J., Dahl D.A. SIMION ${ }^{\mathrm{TM}} 8.0$ User Manual. Sci. Instrument Services, Inc. Idaho Nat. Lab., 2006. 437 p. 